\title{
Resultados de una encuesta sobre donación y trasplante de órganos en la zona metropolitana de Guadalajara, México
}

Luz Consuelo Zepeda-Romero, MC, (1) Guillermo García-García, MC, ,1) O scar A guirre-Jaúregui, MC .11

\section{Zepeda-Romero LC, García-García G,Aguirre-Jaúregui 0. Resultados de una encuesta sobre donación y trasplante de órganos en la zona metropolitana de Guadalajara, México. Salud Publica Mex 2003;45:54-57. \\ El texto completo en inglés de este artículo está disponible en: http://www.insp.mx/salud/index.html}

\section{Resumen}

Objetivo. Evaluar el conocimiento y la disposición de la donación de órganos y tejidos con fines de trasplante entre la población adulta del área metropolitana de Guadalajara, Jalisco, México. Material y métodos. Se realizó una encuesta aplicando un cuestionario de 29 reactivos a personas mayo res de 18 años de edad, mediante entrevista directa en la vía pública. La zona metropolitana se dividió en cuatro municipios, asignándose a cada uno de éstos el número de encuestas en proporción al número de habitantes, estrato socioeconómico, edad y género. Se calcularon frecuencias y $\chi^{2}$ para diferencias de proporciones. Resultados Se encuestaron 400 individuos. El $80.4 \%$ conocía acerca de la donación de órganos y $65 \%$ sabía que está permitido por la ley; sin embargo, solamente $24 \%$ había comentado el tema en familia. El $71 \%$ de los encuestados desconocía cómo donar sus órganos y sólo 25\% sabía de la existencia de la tarjeta de donación. Por otra parte, $66 \%$ estarían dispuestos a donarlos, $16.5 \%$ no lo harían y $17 \%$ estaban indecisos. La mutilación corporal fue la razón principal (40\%) para no donar. El 89\% estarían dispuestos a autorizar la extracción de órganos de un familiar, siempre que éste hubiera manifestado su deseo en vida; de lo contrario, sólo $29 \%$ procederían con la autorización. Finalmente, $65 \%$ consideró la licencia de conducir como un medio adecuado de expresar el deseo de donar y $50 \%$ considera insuficiente la información que sobre el tema se ha difundido por los medios de comunicación. Conclusiones. La mayoría de la población de la zona metropolitana de la ciudad de Guadalajara tiene conoci-

\author{
Zepeda-Romero LC, García-García G,Aguirre-Jaúregui 0. \\ Survey results regarding organ donation and transplant \\ in metropolitan Guadalajara, Mexico. \\ Salud Publica Mex 2003;45:54-57. \\ The English version of this paper \\ is available at: http://www.insp.mx/salud/index.html
}

\begin{abstract}
A bstract
Objective. To evaluate knowledge of and disposition to wards organ and tissue donation for transplantation among the adult population of metropolitan Guadalajara, Jalisco, Mexico. Material and Methods A survey was conducted by personal interview with street-recruited persons over 18 years of age using a 29-item questionnaire. Metro po litan Guadalajara was divided into four municipalities and a proportional number of inter views was assigned to each one based on number of inhabitants, socio-economic stratum, age and gender. Results. Four hundred individuals were interviewed. A pproximately $80 \%$ knew about organ donation and $65 \%$ knew that it was legal; however, only $24 \%$ had discussed the topic with their relatives. Seventyone percent did not know how to donate organs and only $25 \%$ knew about organ donor cards. On the other hand, $66 \%$ were willing to donate, $16.5 \%$ would not donate and $17 \%$ were undecided. Bodily mutilation was the main reason (40\%) given to not donate. $89 \%$ would authorize organ donation from a deceased relative, assuming the relative had previously expressed the desire to donate; on the contrary, only $29 \%$ would give such an authorization without their relative's prior consent. Finally, $65 \%$ considered the driver's license an adequate mean to express their desire to donate and $50 \%$ considered the information the mass media disseminated on the subject to be insufficient. Conclusions. The majority of the metropolitan Guadalajara population knew about organ donation and would be willing to donate their organs. However, a high percentage did not know how
\end{abstract}

(1) Antiguo Hospital C ivil de Guadalajara O P.D. Hospital Civil de Guadalajara, Centro Universitario de Ciencias de la Salud, Universidad de Guadalajara, México.

Fecha de recibido: 13 de marzo de 2002 - Fecha de aprobado: 12 de septiembre de 2002

Solicitud de sobretiros: Dra. Luz Consuelo Zepeda-Romero. Centro Universitario de Ciencias de la Salud. Calle El C armen 305. 45040, Guadalajara, México

Correo electrónico: ConsueloZepeda@ gmx.net 
miento sobre la donación de órganos y estaría dispuesta a donarlos. Sin embargo, un alto porcentaje desconoce el cómo hacerlo y considera insuficiente la información difundida sobre el tema. Una campaña permanente a través de los medios de comunicación masiva podría solucionar lo anterior e incrementaría la donación de órganos en esta población. El texto completo en inglés de este artículo está disponible en: http://www.insp.mx/salud/index.html

Palabras clave: donación de órganos; conocimiento; trasplante; actitud; M éxico to donate and considered the information disseminated on the subject insufficient. An on-going mass media campaign could resolve this matter and hopefully increase organ donation among this population. The English version of this paper is available at: http://www.insp.mx/salud/index.html

Key words: organ donation; knowledge; attitude; transplant; Mexico
E n 1963 se realizó en México el primer trasplante renal, iniciando así una nueva era en la medicina en nuestro país. ${ }^{1}$ En 1968 en el Antiguo Hospital Civil de Guadalajara (AHCG) se efectuó el primer trasplante de córnea y, desde entonces, esta institución ha hecho esfuerzos para concienciar a la población sobre la importancia de la donación de órganos y tejidos. Sin embargo, por el número limitado de donaciones cadavéricas, estos esfuerzos informales parecen no haber producido un impacto significativo en la población. Por otra parte, el aumento en la demanda de trasplantes renales y de córneas, y el desarrollo de programas de trasplante de otros órganos, como hígado y corazón, han incrementado el interés en la donación de órganos en nuestra ciudad y en el país en general. Además, han sido documentadas tanto la necesidad de aumentar la disponibilidad de órganos con fines de trasplante, así como las dificultades encontradas en México en el proceso de procuración de ellos. ${ }^{1,2}$

Reconociendo que las actitudes y conocimientos de la población acerca de la donación de órganos son parte esencial del proceso de su obtención, se procedió a evaluar la percepción y la disposición al respecto entre nuestra población.

\section{Material y métodos}

Se elaboró un cuestionario con 29 reactivos de opción múltiple, con la asesoría del Centro de Estudios de Opinión de la Universidad de Guadalajara, empresa que se responsabilizó de aplicarlo mediante entrevista directa en la vía pública, asegurando el anonimato de los encuestados. El universo de estudio fue la población mayor de 18 años de edad de la zona metropolitana de Guadalajara, la cual comprende los municipios de Guadalajara, Zapopan, Tlaquepaque y Tonalá. La unidad de muestreo fueron las Areas Geoestadísticas Básicas (AGEB), subdivisiones territoriales menores a la división municipal, las cuales facilitan la captación estadística. A cada municipio se le asignó un número de encuestas proporcional a su población. El estrato socioeconómico fue dividido en alto, medio, bajo y marginal, y cada uno de ellos se subdividió en superior e inferior, siguiendo la clasificación del Instituto Nacional de Estadística, Geografía e Informática (INEGI). Finalmente, se asignaron cuotas en cada AGEB, de acuerdo con grupos de edad y género. El marco muestral fue elaborado de acuerdo con un diseño de muestreo aleatorio estratificado de una etapa para proporciones, ${ }^{3}$ y el tamaño de la muestra se determinó para una confiabilidad de 95\%.

\section{Resultados}

Se aplicó el cuestionario en 400 individuos; de éstos $51 \%$ correspondieron al sexo femenino. La distribución por edad y género se muestra en el cuadro I. El 95\% profesaba la religión católica, por lo que los resultados se aplican a esta religión. El 80.4\% conocía acerca de la donación de órganos; sin embargo, sólo 24\% había comentado el tema en familia. De estos últimos la mayoría fueron mujeres (55\%), jóvenes entre 18 y 24 años de edad (28\%) y de un estrato socioeconómico medio superior. El 65\% sabía que la donación está permitida por ley, pero $71 \%$ desconocía el cómo donar sus órganos y sólo $25 \%$ sabía de la existencia de la tarjeta de

Cuadro I

DistRIBUCIÓN DE LA POBLACIÓN POR EDAD Y GÉNERO. Guadalajara, Jalisco, MÉXICO, 1997

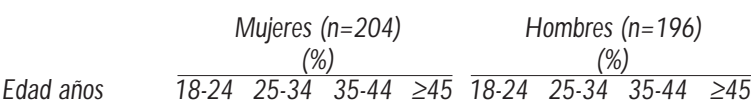

Municipio

\begin{tabular}{lrrrrrrrr} 
Guadalajara & 14 & 14 & 10 & 15 & 13 & 12 & 9 & 13 \\
\hline Tlaquepaque & 14 & 15 & 10 & 12 & 14 & 13 & 10 & 12 \\
\hline Tonalá & 13 & 16 & 9 & 10 & 14 & 17 & 10 & 11 \\
\hline Zapopan & 14 & 16 & 11 & 12 & 13 & 13 & 10 & 11 \\
\hline
\end{tabular}


donación. Entre los que conocían cómo donar sus órganos predominaron las personas jóvenes entre los 18 y 24 años de edad y adultos mayores de 45 años, de estrato socioeconómico bajo superior, sin encontrar diferencias por género (cuadro II).

Por otra parte, $66 \%$ estarían dispuestos a donar sus órganos, mientras que $16.5 \%$ no lo harían y $17 \%$ se mostraron indecisos. De quienes no estarían dispuestos a donar la mayoría fue de hombres, entre $35 \mathrm{y}$ 44 años de edad y de estrato socioeconómico medio inferior (cuadro III). El temor a la mutilación personal fue el motivo principal (40\%) para negarse a donar. En cuanto al hecho de que su familia respetara el deseo de donar órganos a su muerte, $70 \%$ manifestó que sí lo harían y hasta $89 \%$ respetarían a su vez la voluntad de un familiar de donar, siempre que éste lo haya manifestado así; de lo contrario, sólo $29 \%$ autorizarían la donación $(p=0.031)$.

Finalmente, $65 \%$ estarían de acuerdo en manifestar su voluntad en la licencia de conducir, y 50\% consideró insuficiente la información vertida sobre la donación de órganos en nuestra región.

\section{Cuadro II \\ Conocimiento de la población sobre cómo SE PUEDEN DONAR ÓRGANOS PARA TRASPLANTE. DISTRIBUCIÓN POR EDAD, GÉNERO Y ESTRATO SOCIOECONÓMICO. Guadalajara, Jalisco, México, 1997}

$\begin{array}{ll}\text { Sí } & \text { No } \\ (\%) & (\%)\end{array}$

\begin{tabular}{lll}
$\begin{array}{l}\text { Edad (años): } \\
18-24\end{array}$ & 36.4 & 63.6 \\
\hline $25-34$ & 31.0 & 69.0 \\
\hline $35-44$ & 30.9 & 69.1 \\
\hline$\geq 45$ & 38.4 & 61.6
\end{tabular}

Género

\begin{tabular}{lll} 
Femenino & 34.0 & 66.0 \\
\hline Masculino & 35.0 & 65.0
\end{tabular}

Estrato socioeconómico

\begin{tabular}{lll} 
Alto inferior & 35.0 & 65.0 \\
\hline Medio superior & 34.0 & 66.0 \\
\hline Medio inferior & 32.2 & 67.8 \\
\hline Bajo superior & 45.5 & 54.6 \\
\hline Bajo inferior & 32.2 & 67.8 \\
\hline Marginal superior & 25.0 & 75.0
\end{tabular}

\section{Cuadro III \\ DisPosición DE donar ÓRganOS PARA TRASPLANTE. DistRIBUCIÓN POR EDAD, GÉNERO Y ESTRATO SOCIOECONÓMICO. Guadalajara, Jalisco, México, 1997}

$\begin{array}{ccc}\text { Sí } & \text { No } & \text { Indeciso } \\ (\%) & (\%) & (\%)\end{array}$

\begin{tabular}{cccc}
$\begin{array}{c}\text { Edad (años) } \\
18-24\end{array}$ & 76.9 & 10.2 & 13.0 \\
\hline $25-34$ & 72.5 & 11.0 & 16.5 \\
\hline $35-44$ & 56.8 & 27.0 & 16.2 \\
\hline$\geq 45$ & 55.1 & 21.5 & 23.4
\end{tabular}

Género

\begin{tabular}{llll} 
Femenino & 69.1 & 12.6 & 18.4 \\
\hline Masculino & 63.0 & 20.8 & 16.2
\end{tabular}

Estrato socioeconómico

\begin{tabular}{lrrr} 
Alto inferior & 76.0 & 16.0 & 8.0 \\
\hline Medio superior & 81.0 & 6.9 & 12.1 \\
\hline Medio inferior & 58.0 & 25.0 & 17.0 \\
\hline Bajo superior & 52.9 & 17.7 & 17.0 \\
\hline Bajo inferior & 63.3 & 15.1 & 21.7 \\
\hline Marginal superior & 81.0 & 6.9 & 12.1
\end{tabular}

\section{Discusión}

Los resultados de esta encuesta muestran que la mayoría de la población de la zona metropolitana de Guadalajara conoce y apoya la donación de órganos con fines de trasplante, lo cual es similar a lo reportado en otros estudios..$^{5-8}$ Sin embargo, en nuestra ciudad, de acuerdo con lo difundido por el Consejo Estatal de Trasplantes de Organos y Tejidos de Jalisco* sólo se obtienen alrededor de 42 donaciones al año, para una tasa anual de 6.3 donaciones por millón de habitantes, muy por debajo de lo obtenido en otros países. ${ }^{9}$ Las razones son múltiples, sin embargo, destaca el hecho de que un alto número de personas desconoce cómo se pueden donar órganos al morir. Esto pudiera indicar que la información vertida es insuficiente, o bien, que los medios utilizados no han sido los adecuados. Por otra parte, resalta el impacto de la comunicación familiar

\footnotetext{
* Consejo Estatal de Organos y Tejidos/Jalisco. Estadística. http:// www.jalisco.gob.mx/organismos/cetot/consultado el 27 de sep-
} tiembre de 2002 
sobre la decisión de autorizar la extracción de órganos: la mayor parte de la población estaría de acuerdo con autorizar la donación de órganos de un familiar, cuando el fallecido así lo hubiese expresado. También es motivante el hecho de que la voluntad de donar sería respetada. Esta actitud es similar a la encontrada en España. ${ }^{8,10}$ Sin embargo, es preocupante que sólo $25 \%$ trate el tema en familia, ya que en el proceso de donación la ley exige la autorización del familiar más cercano. ${ }^{1,11,12}$ Un paso a seguir sería fomentar la discusión familiar del tema en las campañas de donación. Es motivante la buena disposición de manifestar el deseo de donar en la licencia de conducir, lo cual ha dado buenos resultados en los Estados Unidos de América ${ }^{13}$ y ya ha sido adoptado por nuestra legislación estatal.

En conclusión, este estudio muestra que la mayoría de nuestra población conoce sobre el tema de la donación y que existe una buena disposición para donar. Sin embargo, un alto porcentaje desconoce cómo hacerlo y considera insuficiente la información difundida. Una campaña permanente de donación a través de los medios de comunicación masiva podría solucionar lo anterior y posiblemente incrementaría la donación de órganos en nuestra ciudad. ${ }^{14}$

\section{Referencias}

1. So beranes A, Baltazar S, C ervantes B, Tovar E,Villalobos M, C alderón L et al. Legal basis for cadaver donation in Mexico:A simple diagram that facilitates the legal procedure, initial experience. Transplant Proc 1997; 29:3228-3230

2. Miranda B, Fernández LM, Matnesanz R.The potential organ donor pool: International figures. Transplant Proc 1997;29:1604.

3. Sukhatme PV, Sukhatme BV. Sampling theory of surveys with applications. 3rd edition. Ames: Iowa State Press, 1984:143.

4. Cochran W G. Sampling techniques. 3rd edition. N ueva York (N Y): John W iley and Sons, 1977:75.

5. D o mínguez JM, G onzález ZA, M orales LA, Torres A, Santiago-D elpin E. Knowledge and attitude about organ donation in a Hispanic population. Transplant Proc 1991;23(2):1804-1806.

6. Duarte PS, Pericoco S, Miyazaki MCOS, Ramalho HJ, Abbud-Filho M. Brazilian's attitudes toward organ donation and transplantation.Transplant Proc 2002;34:458-459.

7. Gross T, Martinolis S, Spagnoli G, Badia F, Malacrida R. Attitudes and behaviour of young European adults towards the donation of organs - a call for better information. Am J Transplant 2001;1(1):74-81.

8. Martínez JM, Martín A, López JS. Spanish public opinion concerning organ donation and transplantation. Med Clin 1995;105(11):401-406.

9. Richmond VA, Rockville MD. 2000 Annual report of the U.S. scientific registry of transplant recipients and the organ procurement and transplantation network: Transplant data 1989-1998. (2001, July 6). VA:H HS/ HRSA/O SP/D OT and UN OS. Retrieved (July 22,2002). Available from: URL: http://www.unos.org/frame_D efault.asp?C ategory=anrpt.

10. Martínez JM, López JS, Martín A, Martín MJ, Scandroglio B, Martín JM. 0 rgan donation and family decision-making within the Spanish donation system. Soc Sci Med 2001;53(4):405-421.

11. Dejong W, Franz HG, W olfe SM, N athan H, Payne D, ReitsmaW et al. Requesting organ donation:An interview study of donor and non-donor families. Am J Crit Care 1998;7:13.

12. Poder Ejecutivo Federal. D ecreto por el que se reforma la Ley General de Salud. Estados Unidos Mexicanos: Diario 0 ficial de la Federación, 29 de mayo de 2000.

13. Spital A. Mandated choice. A plan to increase public commitment to organ donation. JAMA 1995;273:504-506.

14. Callender $\mathrm{CO}$,W ashington AW. 0 rgan/tissue donation the problem! education the solution:A review. J N atl Med Assoc 1997:89(10):689-693. 